Article

\title{
Short-Time Propagators and the Born-Jordan Quantization Rule
}

\author{
Maurice A. de Gosson
}

Faculty of Mathematics (NuHAG), University of Vienna, Oskar-Morgenstern-Platz 1, 1090 Vienna, Austria; maurice.de.gosson@univie.ac.at

Received: 14 October 2018; Accepted: 8 November 2018; Published: 10 November 2018

check for updates

\begin{abstract}
We have shown in previous work that the equivalence of the Heisenberg and Schrödinger pictures of quantum mechanics requires the use of the Born and Jordan quantization rules. In the present work we give further evidence that the Born-Jordan rule is the correct quantization scheme for quantum mechanics. For this purpose we use correct short-time approximations to the action functional, initially due to Makri and Miller, and show that these lead to the desired quantization of the classical Hamiltonian.
\end{abstract}

Keywords: Born-Jordan quantization; short-time propagators; time-slicing; Van Vleck determinant

\section{Motivation and Background}

\subsection{Weyl versus Born and Jordan}

There have been several attempts in the literature to find the "right" quantization rule for observables using either algebraic or analytical techniques [1-7]. In a recent paper [8] we have analyzed the Heisenberg and Schrödinger pictures of quantum mechanics, and shown that if one postulates that both theories are equivalent, then one must use the Born-Jordan quantization rule

$$
\text { (BJ) } \quad x^{m} p^{\ell} \longrightarrow \frac{1}{m+1} \sum_{k=0}^{m} \widehat{x}^{k} \hat{p}^{\ell} \widehat{x}^{m-k}
$$

and not the Weyl rule (To be accurate, it was McCoy [9] who showed that Weyl's quantization scheme leads to Formula (2)).

$$
\text { (Weyl) } \quad x^{m} p^{\ell} \longrightarrow \frac{1}{2^{m}} \sum_{k=0}^{m}\left(\begin{array}{c}
m \\
k
\end{array}\right) \widehat{x}^{k} \hat{p}^{\ell} \widehat{x}^{m-k}
$$

for monomial observables. The Born-Jordan and Weyl rules yield the same result only if $m<2$ or $\ell<2$; for instance in both cases the quantization of the product $x p$ is $\frac{1}{2}(\widehat{x} \widehat{p}+\widehat{p} \widehat{x})$. One can also show that the product $p f(x)$ is, for any smooth function $f$ of position alone, given in both cases by the symmetric rule

$$
p f(x) \longrightarrow \frac{1}{2}(\hat{p} f(x)+f(x) \widehat{p}) .
$$

It follows that if $H$ is a Hamiltonian of the type

$$
H=\sum_{j=1}^{n} \frac{1}{2 m_{j}}\left(p_{j}-A_{j}(x)\right)^{2}+V(x)
$$

one can use either the Weyl or the Born-Jordan prescriptions to get the the corresponding quantum operator, which yields the familiar expression 


$$
\widehat{H}=\sum_{j=1}^{n} \frac{1}{2 m_{j}}\left(-i \hbar \frac{\partial}{\partial x_{j}}-A(x)\right)^{2}+V(x) .
$$

(See Section 3.3). Since this Hamiltonian is without doubt the one which most often occurs in quantum mechanics one could ask why one should bother about which is the "correct" quantization. It turns out that this question is just a little bit more than academic: There are simple physical observables which yield different quantizations in the Weyl and Born-Jordan schemes. One interesting example is that of the squared angular momentum: Writing $\mathbf{r}=(x, y, z)$ and $\mathbf{p}=\left(p_{x}, p_{y}, p_{z}\right)$ the square of the classical angular momentum

$$
\ell=\left(y p_{z}-z p_{y}\right) \mathbf{i}+\left(z p_{x}-x p_{z}\right) \mathbf{j}+\left(x p_{y}-y p_{x}\right) \mathbf{k}
$$

is the function $\ell^{2}=\ell_{x}^{2}+\ell_{y}^{2}+\ell_{z}^{2}$ where

$$
\ell_{x}^{2}=x^{2} p_{y}^{2}+y^{2} p_{x}^{2}-2 x p_{x} y p_{y}
$$

and so on. The Weyl quantization of $\ell_{x}^{2}$ is

$$
\left(\widehat{\ell}_{x}^{2}\right)_{\mathrm{W}}=\widehat{x}^{2} \hat{p}_{y}^{2}+\widehat{x}_{y}^{2} \hat{p}_{x}^{2}-\frac{1}{2}\left(\widehat{x} \widehat{p}_{x}+\hat{p}_{x} \widehat{x}\right)\left(\widehat{y} \widehat{p}_{y}+\widehat{p}_{y} \widehat{y}\right)
$$

while its Born-Jordan quantization is

$$
\left(\widehat{\ell}_{x}^{2}\right)_{\text {BJ }}=\widehat{x}^{2} \widehat{p}_{y}^{2}+\widehat{x}_{y}^{2} \widehat{p}_{x}^{2}-\frac{1}{2}\left(\widehat{x} \hat{p}_{x}+\widehat{p}_{x} \widehat{x}\right)\left(\widehat{y} \widehat{p}_{y}+\widehat{p}_{y} \widehat{y}\right)-\frac{1}{6} \hbar^{2} ;
$$

similar relations are obtained for $\ell_{y}^{2}$ and $\ell_{z}^{2}$ so that, in the end,

$$
\left(\widehat{\ell^{2}}\right)_{\mathrm{W}}-\left(\widehat{\ell^{2}}\right)_{\mathrm{BJ}}=\frac{1}{2} \hbar^{2}
$$

This discrepancy has been dubbed the "angular momentum dilemma "[10]; in [11] we have discussed this apparent paradox and shown that it disappears if one systematically uses Born-Jordan quantization.

\subsection{The Kerner and Sutcliffe Approach to Quantization}

As we have proven in [8,12], Heisenberg's matrix mechanics [13], as rigorously constructed by Born and Jordan in [14] and Born, Jordan, and Heisenberg in [15], explicitly requires the use of the quantization rule (1) to be mathematically consistent, a fact which apparently has escaped the attention of physicists, and philosophers or historians of Science. In the present paper, we will show that the Feynman path integral approach is another genuinely physical motivation for Born-Jordan quantization of arbitrary observables; it corrects previous unsuccessful attempts involving path integral arguments which do not work for a reason that will be explained. One of the most convincing of these attempts is the paper [16] by Kerner and Sutcliffe. Elaborating on previous work of Garrod [17] Kerner and Sutcliffe tried to justify the Born-Jordan rule as the unique possible quantization (see Steven Kauffmann's [18,19] brilliant discussion of this work). Assuming that $\hat{H}$ is the quantization of some general Hamiltonian $H$, they write as is usual in the theory of the phase space Feynman integral the propagator as

$$
\left\langle x\left|e^{-\frac{i}{\hbar} \widehat{H} t}\right| x^{\prime}\right\rangle=\lim _{N \rightarrow \infty} \int d x_{N-1} \cdots d x_{1} \prod_{k=1}^{N}\left\langle x_{k}\left|e^{-\frac{i}{\hbar} \widehat{H} \Delta t}\right| x_{k-1}\right\rangle
$$

where $x_{N}=x$ and $x_{0}=x^{\prime}$ are fixed and $\Delta t=t / N$. They thereafter use the approximation

$$
\left\langle x_{k}\left|e^{-\frac{i}{\hbar} \widehat{H} \Delta t}\right| x_{k-1}\right\rangle \approx \frac{1}{2 \pi \hbar} \int e^{\frac{i}{\hbar} \bar{S}\left(x, x^{\prime}, p, \Delta t\right)} d p
$$


the function $\bar{S}$ being given by

$$
\bar{S}\left(x, x^{\prime}, p, \Delta t\right)=p\left(x-x^{\prime}\right)-\bar{H}\left(x, x^{\prime}, p\right) \Delta t
$$

where $\bar{H}$ is the time average of $H$ over $p$ fixed and $x=x(t)$, that is

$$
\bar{H}\left(x, x^{\prime}, p\right)=\frac{1}{\Delta t} \int_{0}^{\Delta t} H\left(x^{\prime}+s \frac{x-x^{\prime}}{\Delta t}, p\right) d s .
$$

Notice that introducing the dimensionless parameter $\tau=s / \Delta t$, Formula (11) can be written in the more convenient form

$$
\bar{H}\left(x, x^{\prime}, p\right)=\int_{0}^{1} H\left(\tau x+(1-\tau) x^{\prime}, p\right) d \tau
$$

which is the usual mathematical definition of Born-Jordan quantization: See de Gosson $[12,20]$ and de Gosson and Luef [21].

Taking the limit $\Delta t \rightarrow 0$ the operator $\widehat{H}$ can then be explicitly and uniquely determined, and Kerner and Sutcliffe show that in particular this leads to the Born-Jordan ordering (1) when their Hamiltonian $H$ is a monomial $x^{m} p^{\ell}$. Unfortunately (as immediately Cohen's rebuttal was published in the same volume of J. Math. Phys. in which Kerner and Sutcliffe published their results. Noted by Cohen [22]) there are many a priori equally good constructions of the Feynman integral, leading to other quantization rules. In fact, argues Cohen, there is a great freedom of choice in calculating the action $p\left(x-x^{\prime}\right)-\bar{H}$ appearing in the right-hand side of (11). For instance, one can choose

$$
S\left(x, x^{\prime}, p, \Delta t\right)=p\left(x-x^{\prime}\right)-H\left(\frac{1}{2}\left(x+x^{\prime}\right), p\right) \Delta t
$$

which leads for $x^{m} p^{\ell}$ to Weyl's rule (2), or one can choose

$$
S\left(x, x^{\prime}, p, \Delta t\right)=p\left(x-x^{\prime}\right)-\frac{1}{2}\left(H(x, p)+H\left(x^{\prime}, p\right)\right) \Delta t,
$$

which leads to the symmetric rule

$$
x^{m} p^{\ell} \longrightarrow \frac{1}{2}\left(\widehat{x}^{m} \widehat{p}^{\ell}+\widehat{p}^{\ell} \widehat{x}^{m}\right) .
$$

This ambiguity shows-in an obvious way-that Feynman path integral theory does not lead to an uniquely defined quantization scheme for observables. However-and this is the main point of the present paper-while Cohen's remark was mathematically justified, Kerner and Sutcliffe's insight was right (albeit for the wrong reason).

\subsection{What We Will Do}

It turns out that the Formula (10) for the approximate action that Kerner and Sutcliffe "guessed" has been justified independently (in another context) by Makri and Miller $[23,24]$ and the present author [25] by rigorous mathematical methods. This formula is actually the correct approximation to action up to order $O\left(\Delta t^{2}\right)$ (as opposed to the "midpoint rules" commonly used in the theory of the Feynman integral which yield much cruder approximations); it follows that Kerner and Sutcliffe's Formula (9) indeed yields a correct approximation of the infinitesimal propagator $\left\langle x_{k}\left|e^{-\frac{i}{\hbar} \widehat{H} \Delta t}\right| x_{k-1}\right\rangle$, in fact the best one for calculational purposes since it ensures a swift convergence of numerical schemes. This is because for short times $\Delta t$ the solution of Schrödinger's equation

$$
i \hbar \frac{\partial \psi}{\partial t}(x, t)=\left[\sum_{j=1}^{n} \frac{-\hbar^{2}}{2 m_{j}} \frac{\partial^{2}}{\partial x_{j}^{2}}+V(x)\right] \psi(x, t)
$$


with initial condition $\psi(x, 0)=\psi_{0}(x)$ is given by the asymptotic formula

$$
\psi(x, \Delta t)=\int \bar{K}\left(x, x^{\prime}, \Delta t\right) \psi_{0}\left(x^{\prime}\right) d^{n} x^{\prime}+O\left(\Delta t^{2}\right) ;
$$

the approximate propagator $\bar{K}$ being defined, for arbitrary time $t$, by

$$
\bar{K}\left(x, x^{\prime}, t\right)=\left(\frac{1}{2 \pi \hbar}\right)^{n} \int \exp \left(\frac{i}{\hbar}\left[p\left(x-x^{\prime}\right)-\left(H_{\text {free }}(p)+\bar{V}\left(x, x^{\prime}\right)\right) t\right]\right) d^{n} p ，
$$

where, by definition, $H_{\text {free }}(p)$ is the free particle Hamiltonian function, and the two-point function

$$
\bar{V}\left(x, x^{\prime}\right)=\int_{0}^{1} V\left(\tau x+(1-\tau) x^{\prime}\right) d \tau
$$

is the average value of the potential $V$ on the line segment $\left[x^{\prime}, x\right]$.

- In Section 2 we discuss the accuracy of Kerner and Sutcliffe's propagator by comparing it with the more familiar Van Vleck propagator; we show that for small times both are approximations to order $O\left(t^{2}\right)$ to the exact propagator of Schrödinger's equation.

- In Section 3 we show that if one assume's that short-time evolution of the wavefunction (for an arbitrary Hamiltonian $H$ ) is given by the Kerner and Sutcliffe propagator, then $H$ must be quantized following the rule (12); we thereafter show that when $H$ is a monomial $x^{m} p^{\ell}$ then the corresponding operator is given by the Born-Jordan rule (1), not by the Weyl rule 2.

Notation 1. The generalized position and momentum vectors are $x=\left(x_{1}, \ldots, x_{n}\right)$ and $p=\left(p_{1}, \ldots, p_{n}\right)$; we set $p x=p_{1} x_{1}+\cdots+p_{n} x_{n}$. We denote by $\widehat{x}_{j}$ the operator of multiplication by $x_{j}$ and by $\hat{p}_{j}$ the momentum operator $-i \hbar\left(\partial / \partial x_{j}\right)$.

\section{On Short-Time Propagators}

In this section we only consider Hamiltonian functions of the type "kinetic energy plus potential":

$$
H(x, p)=H_{\text {free }}(p)+V(x), H_{\text {free }}(p)=\sum_{j=1}^{n} \frac{1}{2 m_{j}} p_{j}^{2} .
$$

These are the simplest physical Hamiltonians, both from a classical and a quantum perspective.

\subsection{The Van Vleck Propagator}

Consider a Hamiltonian function of the type (19) above; the corresponding Schrödinger equation is

$$
i \hbar \frac{\partial \psi}{\partial t}(x, t)=\left[\sum_{j=1}^{n} \frac{-\hbar^{2}}{2 m_{j}} \frac{\partial^{2}}{\partial x_{j}^{2}}+V(x)\right] \psi(x, t) .
$$

We will denote by $K\left(x, x^{\prime}, t\right)=\left\langle x\left|e^{-\frac{i}{\hbar} \hat{H} t}\right| x^{\prime}\right\rangle$ the corresponding exact propagator:

$$
\psi(x, t)=\int K\left(x, x^{\prime}, t\right) \psi_{0}\left(x^{\prime}\right) d^{n} x^{\prime}
$$

where with $\psi_{0}(x)$ is the value of $\psi$ at time $t=0$. The function $K\left(x, x^{\prime}, t\right)$ must thus satisfy the boundary condition

$$
\lim _{t \rightarrow 0} K\left(x, x^{\prime}, t\right)=\delta\left(x-x^{\prime}\right)
$$


It is well-known (see e.g., Gutzwiller [26], Schulman [27], de Gosson [25], Maslov and Fedoriuk [28]) that for short times an approximate propagator is given by Van Vleck's formula

$$
\widetilde{K}\left(x, x^{\prime}, t\right)=\left(\frac{1}{2 \pi i \hbar}\right)^{n / 2} \sqrt{\rho\left(x, x^{\prime}, t\right)} e^{\frac{i}{\hbar} S\left(x, x^{\prime}, t\right)}
$$

where

$$
S\left(x, x^{\prime}, t\right)=\int_{0}^{t}\left(\sum_{j=1}^{n} \frac{1}{2} m_{j} \dot{x}_{j}(s)^{2}-V(x(s)) d s\right.
$$

is the action along the classical trajectory leading from $x^{\prime}$ at time $t^{\prime}=0$ to $x$ at time $t$ (there is no sum over different classical trajectories because only one trajectory contributes in the limit $t \rightarrow 0$ [23]) and

$$
\rho\left(x, x^{\prime}, t\right)=\operatorname{det}\left(-\frac{\partial^{2} S\left(x, x^{\prime}, t\right)}{\partial x_{j} \partial x_{j k}^{\prime}}\right)_{1 \leq j, k \leq n}
$$

is the Van Vleck density of trajectories [25-27]; the argument of the square root is chosen so that the initial condition (22) is satisfied [25,29]. It should be emphasized that although the Van Vleck propagator is frequently used in semiclassical mechanics, it has nothing "semiclassical" per se, since it is genuinely an approximation to the exact propagator for small $t$ - not just in the limit $\hbar \rightarrow 0$. In fact:

Theorem 1. Let $\widetilde{\psi}$ be given by

$$
\widetilde{\psi}(x, t)=\int \widetilde{K}\left(x, x^{\prime}, t\right) \psi_{0}\left(x^{\prime}\right) d^{n} x^{\prime}
$$

where $\psi_{0}$ is a tempered distribution. Let $\psi$ be the exact solution of Schrödinger's equation with initial datum $\psi_{0}$. We have

$$
\psi(x, t)-\widetilde{\psi}(x, t)=O\left(t^{2}\right) .
$$

In particular, the Van Vleck propagator $\widetilde{K}\left(x, x^{\prime}, t\right)$ is an $O\left(t^{2}\right)$ approximation to the exact propagator $K\left(x, x^{\prime}, t\right)$ :

$$
K\left(x, x^{\prime}, t\right)-\widetilde{K}\left(x, x^{\prime}, t\right)=O\left(t^{2}\right)
$$

for $t \rightarrow 0$ and hence

$$
\lim _{t \rightarrow 0} \widetilde{K}\left(x, x^{\prime}, t\right)=\delta\left(x-x^{\prime}\right) .
$$

Proof. Referring to de Gosson [25] (Lemma 241) for details, we sketch the main lines in the case $n=1$. Assuming that $\psi_{0}$ belongs to the Schwartz space $\mathcal{S}\left(\mathbb{R}^{n}\right)$ of rapidly decreasing functions, one expands the solution $\psi$ of Schrödinger's equation to second order:

$$
\psi(x, t)=\psi_{0}(x)+\frac{\partial \psi}{\partial t}(x, 0) t+O\left(t^{2}\right) .
$$

Taking into account the fact that $\psi$ is a solution of Schrödinger's equation this can be rewritten

$$
\psi(x, t)=\left[1+\frac{t}{i \hbar}\left(-\frac{\hbar^{2}}{2 m} \frac{\partial^{2}}{\partial x^{2}}+V(x)\right)\right] \psi_{0}(x)+O\left(t^{2}\right) .
$$

Expanding the exponential $e^{i S / \hbar}$ in Van Vleck's Formula (23) at $t=0$ one shows, using the estimate (32) in Theorem 2, that we also have

$$
\widetilde{\psi}(x, t)=\left[1+\frac{t}{i \hbar}\left(-\frac{\hbar^{2}}{2 m} \frac{\partial^{2}}{\partial x^{2}}+V(x)\right)\right] \psi_{0}(x)+O\left(t^{2}\right)
$$


comparison with (28) implies that $\psi(x, t)-\widetilde{\psi}(x, t)=O\left(t^{2}\right)$. By density of the Schwartz space in the class of tempered distributions $\mathcal{S}^{\prime}\left(\mathbb{R}^{n}\right)$ the estimate (26) is valid if one chooses $\psi_{0}(x)=\delta\left(x-x_{0}\right)$, which yields Formula (27) since we have

$$
\int \widetilde{K}\left(x, x^{\prime}, t\right) \delta\left(x-x_{0}\right) d^{n} x^{\prime}=\widetilde{K}\left(x, x_{0}, t\right)
$$

and

$$
\int K\left(x, x^{\prime}, t\right) \delta\left(x-x_{0}\right) d^{n} x^{\prime}=K\left(x, x_{0}, t\right)
$$

Let us briefly return to the path integral. Replacing the terms $\left\langle x_{k}\left|e^{-\frac{i}{\hbar} \hat{H} \Delta t}\right| x_{k-1}\right\rangle$ in the product Formula (8) with $\widetilde{K}\left(x_{k-1}, x_{k-1}, \Delta t\right)$ one shows, using the Lie-Trotter Formula [25,27], that the exact propagator $K\left(x, x^{\prime}, t\right)=\left\langle x\left|e^{-\frac{i}{\hbar} \hat{H} t}\right| x^{\prime}\right\rangle$ is given by

$$
\left\langle x\left|e^{-\frac{i}{\hbar} \widehat{H} t}\right| x^{\prime}\right\rangle=\lim _{N \rightarrow \infty} \int d x_{N-1} \cdots d x_{1} \prod_{k=1}^{N} \widetilde{K}\left(x_{k-1}, x_{k-1}, \Delta t\right) .
$$

This formula is often taken as the starting point of path integral arguments: observing that the expression (23) is in most cases (The free particle and the harmonic oscillator are remarkable particular cases where the action integral can be explicitly calculated and thus yields an explicit formula for the propagator, but mathematically speaking this fact is rather a consequence of the theory of the metaplectic group [25,29]) difficult to calculate (it implies the computation of an action integral, which can be quite cumbersome) people working in the theory of the Feynman integral replace the exact action $S\left(x, x^{\prime}, t\right)$ in (23) with approximate expressions, for instance the "midpoint rules" that will be discussed below. Now, one should be aware that this legerdemain works, because when taking the limit $N \rightarrow \infty$ one indeed obtains the correct propagator, but it does not imply that these midpoint rules are accurate approximations to $S\left(x, x^{\prime}, t\right)$.

\subsection{The Kerner-Sutcliffe Propagator}

We showed above that the Van Vleck propagator is an approximation to order $O\left(t^{2}\right)$ to the exact propagator. We now show that the propagator proposed by Kerner and Sutcliffe in [16] approximates the Van Vleck propagator also at order $O\left(t^{2}\right)$. Hence

$$
\text { Van Vleck }=\text { Kerner-Sutcliffe }+O\left(t^{2}\right) .
$$

We begin by giving a correct short-time approximation to the action.

Theorem 2. The function $\bar{S}$ defined by

$$
\bar{S}\left(x, x^{\prime}, t\right)=\sum_{j=1}^{n} m_{j} \frac{\left(x_{j}-x_{j}^{\prime}\right)^{2}}{2 t}-\bar{V}\left(x, x^{\prime}\right) t
$$

where $\bar{V}\left(x, x^{\prime}\right)$ is the average of the potential $V$ along the line segment $\left[x^{\prime}, x\right]$ :

$$
\bar{V}\left(x, x^{\prime}\right)=\int_{0}^{1} V\left(\tau x+(1-\tau) x^{\prime}\right) d \tau .
$$

satisfies for $t \rightarrow 0$ the estimate

$$
S\left(x, x^{\prime}, t\right)-\bar{S}\left(x, x^{\prime}, t\right)=O\left(t^{2}\right) .
$$

For detailed proofs we refer to the aforementioned papers $[23,24]$ by Makri and Miller, and to our book [25]; also see de Gosson and Hiley [30,31]. The underlying idea is quite simple (and already 
appears in germ in Park's book [32], p. 438): one remarks that the function $S=S\left(x, x^{\prime}, t\right)$ satisfies the Hamilton-Jacobi equation

$$
\frac{\partial S}{\partial t}+\sum_{j=1}^{n} \frac{1}{2 m_{j}}\left(\frac{\partial S}{\partial x_{j}}\right)^{2}+V(x)=0
$$

and one thereafter looks for an asymptotic solution

$$
S\left(x, x^{\prime}, t\right)=\frac{1}{t} S_{0}\left(x, x^{\prime}\right)+S_{1}\left(x, x^{\prime}\right) t+S_{2}\left(x, x^{\prime}\right) t^{2}+\cdots .
$$

Insertion in (33) then leads to

$$
S_{0}\left(x, x^{\prime}\right)=\sum_{j=1}^{n} m_{j} \frac{\left(x_{j}-x_{j}^{\prime}\right)^{2}}{2}
$$

and $S_{1}\left(x, x^{\prime}\right)=-\bar{V}\left(x, x^{\prime}\right)$ hence (31). Notice that this procedure actually allows one to find approximations to $S$ to an arbitrary order of accuracy by solving successively the equations satisfied by $S_{2}, S_{3}, \ldots$ (see $[23,24]$ for explicit formulas).

Let us now set

$$
\bar{H}\left(x, x^{\prime}, t\right)=H_{\text {free }}(p)+\bar{V}\left(x, x^{\prime}\right)
$$

where

$$
\bar{V}\left(x, x^{\prime}\right)=\int_{0}^{1} V\left(\tau x+(1-\tau) x^{\prime}\right) d \tau
$$

is the averaged potential.

Let us now show that the propagator postulated by Garrod [17] and Kerner and Sutcliffe [16] is as good an approximation to the exact propagator as Van Vleck's is. We recall the textbook Fourier formula

$$
\left(\frac{1}{2 \pi \hbar}\right)^{n} \int e^{\frac{i}{\hbar} p\left(x-x^{\prime}\right)} p_{j}^{\ell} d^{n} p=\left(-i \hbar \frac{\partial}{\partial x_{j}}\right)^{\ell} \delta\left(x-x^{\prime}\right) .
$$

Theorem 3. Let $\bar{K}=\bar{K}\left(x, x^{\prime}, t\right)$ be defined (in the distributional sense) by

$$
\bar{K}\left(x, x^{\prime}, t\right)=\left(\frac{1}{2 \pi \hbar}\right)^{n} \int e^{\frac{i}{\hbar}\left(p\left(x-x^{\prime}\right)-\bar{H}\left(x, x^{\prime}, p\right) t\right)} d^{n} p .
$$

and set

$$
\bar{\psi}(x, t)=\int \bar{K}\left(x, x^{\prime}, t\right) \psi_{0}\left(x^{\prime}\right) d^{n} x^{\prime} .
$$

Let $\psi$ be the solution of Schrödinger's equation with initial condition $\psi_{0}$. We have

$$
\bar{\psi}(x, t)-\psi(x, t)=O\left(t^{2}\right) .
$$

The function $\bar{K}$ is an $O\left(t^{2}\right)$ approximation to the exact propagator $K$ :

$$
K\left(x, x^{\prime}, t\right)-\bar{K}\left(x, x^{\prime}, t\right)=O\left(t^{2}\right) .
$$

Proof. It is sufficient to prove (37); Formula (38) follows by the same argument as in the proof of Theorem 1 . To simplify notation we assume again $n=1$; the general case is a straightforward extension. Expanding for small $t$ the exponential in the integrand of (35) we have

$$
\begin{aligned}
\bar{K}\left(x, x^{\prime}, t\right) & =\left(\frac{1}{2 \pi \hbar}\right)^{n} \int e^{\frac{i}{\hbar} p\left(x-x^{\prime}\right)}\left(1-\frac{i}{\hbar} \bar{H}\left(x, x^{\prime}, p\right) t\right) d p+O\left(t^{2}\right) \\
& =\delta\left(x-x^{\prime}\right)-\frac{i t}{\hbar} \int e^{\frac{i}{\hbar} p\left(x-x^{\prime}\right)} \bar{H}\left(x, x^{\prime}, p\right) d p+O\left(t^{2}\right)
\end{aligned}
$$


and hence

$$
\bar{\psi}(x, t)=\psi_{0}(x)-\frac{i t}{\hbar} \int e^{\frac{i}{\hbar} p\left(x-x^{\prime}\right)} \bar{H}\left(x, x^{\prime}, p\right) \psi_{0}\left(x^{\prime}\right) d p d x^{\prime}+O\left(t^{2}\right) .
$$

We have

$$
\int e^{\frac{i}{\hbar} p\left(x-x^{\prime}\right)} \bar{H}\left(x, x^{\prime}, p\right) d^{n} p=\int e^{\frac{i}{\hbar} p\left(x-x^{\prime}\right)}\left(\frac{p^{2}}{2 m}+\bar{V}\left(x, x^{\prime}\right)\right) d p ;
$$

using the Fourier Formula (34) we get

$$
\left(\frac{1}{2 \pi \hbar}\right)^{n} \int e^{\frac{i}{\hbar} p\left(x-x^{\prime}\right)} \frac{p^{2}}{2 m} d p=-\frac{\hbar^{2}}{2 m} \frac{\partial^{2}}{\partial x^{2}} \delta\left(x-x^{\prime}\right)
$$

and, noting that $\bar{V}(x, x)=V(x)$,

$$
\begin{aligned}
\left(\frac{1}{2 \pi \hbar}\right)^{n} \int e^{\frac{i}{\hbar} p\left(x-x^{\prime}\right)} \bar{V}\left(x, x^{\prime}\right) d p & =\bar{V}\left(x, x^{\prime}\right) \delta\left(x-x^{\prime}\right) \\
& =V(x) \delta\left(x-x^{\prime}\right) .
\end{aligned}
$$

Summarizing,

$$
\bar{K}\left(x, x^{\prime}, t\right)=\delta\left(x-x^{\prime}\right)+\frac{i t}{\hbar}\left(-\frac{\hbar^{2}}{2 m} \frac{\partial^{2}}{\partial x^{2}}+V(x)\right) \delta\left(x-x^{\prime}\right)+O\left(t^{2}\right)
$$

and hence

$$
\bar{\psi}(x, t)=\psi_{0}(x)-\frac{i t}{\hbar}\left(-\frac{\hbar^{2}}{2 m} \frac{\partial^{2}}{\partial x^{2}}+V(x)\right) \psi_{0}(x)+O\left(t^{2}\right) .
$$

Comparing this expression with (28) yields (38).

\subsection{Comparison of Short-Time Propagators}

We have seen above that both the Van Vleck and the Kerner-Sutcliffe propagators are accurate to order $O\left(t^{2}\right)$ :

$$
\begin{aligned}
& K\left(x, x^{\prime}, t\right)-\widetilde{K}\left(x, x^{\prime}, t\right)=O\left(t^{2}\right) . \\
& K\left(x, x^{\prime}, t\right)-\bar{K}\left(x, x^{\prime}, t\right)=O\left(t^{2}\right)
\end{aligned}
$$

and hence, of course,

$$
\widetilde{K}\left(x, x^{\prime}, t\right)-\bar{K}\left(x, x^{\prime}, t\right)=O\left(t^{2}\right) .
$$

Let us now study the case of the most commonly approximations to the action used in the theory of the Feynman integral, namely the mid-point rules

$$
S_{1}\left(x, x^{\prime}, t, t^{\prime}\right)=\sum_{j=1}^{n} m_{j} \frac{\left(x_{j}-x_{j}^{\prime}\right)^{2}}{2 t}-\frac{1}{2}\left(V(x)+V\left(x^{\prime}\right)\right) t
$$

and

$$
S_{2}\left(x, x^{\prime}, t\right)=\sum_{j=1}^{n} m_{j} \frac{\left(x_{j}-x_{j}^{\prime}\right)^{2}}{2 t}-V\left(\frac{1}{2}\left(x+x^{\prime}\right)\right) \Delta t .
$$

We begin with a simple example, that of the harmonic oscillator

$$
H(x, p)=\frac{p^{2}}{2 m}+\frac{1}{2} m^{2} \omega^{2} x^{2}
$$


(we are assuming $n=1$ ). The exact value of the action is given by the generating function

$$
S\left(x, x^{\prime}, t\right)=\frac{m}{2 \sin \omega t}\left(\left(x^{2}+x^{\prime 2}\right) \cos \omega t-2 x x^{\prime}\right) ;
$$

expanding the terms $\sin \omega t$ and $\cos \omega t$ in Taylor series for $t \rightarrow 0$ yields the approximation

$$
S\left(x, x^{\prime}, t\right)=m \frac{\left(x-x^{\prime}\right)^{2}}{2 t}-\frac{m \omega^{2}}{6}\left(x^{2}+x x^{\prime}+x^{\prime 2}\right) t+O\left(t^{2}\right) .
$$

It is easy to verify, averaging $\frac{1}{2} m^{2} \omega^{2} x^{2}$ over $\left[x^{\prime}, x\right]$ that

$$
\bar{S}\left(x, x^{\prime}, t\right)=m \frac{\left(x-x^{\prime}\right)^{2}}{2 t}-\frac{m \omega^{2}}{6}\left(x^{2}+x x^{\prime}+x^{\prime 2}\right) t
$$

is precisely the approximate action provided by (31). If we now instead apply the midpoint rule (43) we get

$$
S_{1}\left(x, x^{\prime}, t\right)=m \frac{\left(x-x^{\prime}\right)^{2}}{2 t}-\frac{m^{2} \omega^{2}}{4}\left(x^{2}+x^{\prime 2}\right) t
$$

which differs from the correct value (46) by a term $O(\Delta t)$. Similarly, the rule (44) yields

$$
S_{2}\left(x, x^{\prime}, t\right)=m \frac{\left(x-x^{\prime}\right)^{2}}{2 t}-\frac{m^{2} \omega^{2}}{8}\left(x+x^{\prime}\right)^{2} t
$$

which again differs from the correct value (45) by a term $O(t)$. It is easy to understand why it is so by examining the case of a general potential function, and to compare $\bar{V}\left(x, x^{\prime}\right), \frac{1}{2}\left(V(x)+V\left(x^{\prime}\right)\right)$, and $V\left(\frac{1}{2}\left(x+x^{\prime}\right)\right.$. Consider for instance $\bar{V}\left(x, x^{\prime}\right)-V\left(\frac{1}{2}\left(x+x^{\prime}\right)\right.$. Expanding $V(x)$ in a Taylor series at $\bar{x}=\frac{1}{2}\left(x+x^{\prime}\right)$ we get after some easy calculations

$$
\begin{aligned}
\bar{V}\left(x, x^{\prime}\right) & =V(\bar{x})+V^{\prime}(\bar{x})\left(x-x^{\prime}\right)+\frac{1}{2} V^{\prime \prime}(\bar{x})\left(x-x^{\prime}\right)^{2}+O\left(\left(x-x^{\prime}\right)^{3}\right) \\
& =V\left(\frac{1}{2}\left(x+x^{\prime}\right)-\frac{1}{12} V^{\prime \prime}\left(\frac{1}{2}\left(x+x^{\prime}\right)\right)\left(x-x^{\prime}\right)^{3}+O\left(\left(x-x^{\prime}\right)^{3}\right)\right.
\end{aligned}
$$

hence $\bar{V}\left(x, x^{\prime}\right)-V\left(\frac{1}{2}\left(x+x^{\prime}\right)\right.$ is different from zero unless $x=x^{\prime}$ (or if $V(x)$ is linear) and hence the difference between $\bar{S}\left(x, x^{\prime}, t\right)$ and $S_{2}\left(x, x^{\prime}, t\right)$ will always generate a term containing $t$ so that $\bar{S}\left(x, x^{\prime}, t\right)-S_{2}\left(x, x^{\prime}, t\right)=O(t)$ (and not $O\left(t^{2}\right)$ ). A similar calculation shows that we will also always have $\bar{S}\left(x, x^{\prime}, t\right)-S_{1}\left(x, x^{\prime}, t\right)=O(t)$. Denoting by $K_{1}\left(x, x^{\prime}, t\right)$ and $K_{2}\left(x, x^{\prime}, t\right)$ the approximate propagators obtained from the midpoint rules (43) and (44), respectively, one checks without difficulty that we will have

$$
\begin{aligned}
& \bar{K}\left(x, x^{\prime}, t\right)-K_{1}\left(x, x^{\prime}, t\right)=O(t) \\
& \bar{K}\left(x, x^{\prime}, t\right)-K_{2}\left(x, x^{\prime}, t\right)=O(t)
\end{aligned}
$$

where $\bar{K}\left(x, x^{\prime}, t\right)$ is the Kerner-Sutcliffe propagator (35) (in these relations we can of course replace $\bar{K}\left(x, x^{\prime}, t\right)$ with the van Vleck propagator $\widetilde{K}\left(x, x^{\prime}, t\right)$ since both differ by a quantity $O\left(t^{2}\right)$ in view of Theorem 3.

\section{The Case of Arbitrary Hamiltonians}

\subsection{The Main Result}

We now consider the following very general situation: We assume that we are in the presence of a quantum system represented by a state $|\psi\rangle$ whose evolution is governed by a strongly continuous one-parameter group $\left(U_{t}\right)$ of unitary operators acting on $L^{2}\left(\mathbb{R}^{n}\right)$; the operator $U_{t}$ takes an initial wavefunction $\psi_{0}$ to $\psi=U_{t} \psi_{0}$. It follows from Schwartz's kernel theorem [33] that there exists a function 
$K=K\left(x, x^{\prime} ; t\right)$ such that (This equality is sometimes postulated; it is in fact a mathematical fact which is true in quite general situations.)

$$
\psi(x, t)=\int K\left(x, x^{\prime} ; t\right) \psi_{0}\left(x^{\prime}\right) d^{n} x^{\prime}
$$

and from Stone's [34] theorem one strongly continuous one-parameter groups of unitary operators that there exists a self-adjoint (generally unbounded) operator $\hat{H}$ on $L^{2}\left(\mathbb{R}^{n}\right)$ such that

$$
\psi(x, t)=e^{-\frac{i}{\hbar} \widehat{H} t} \psi_{0}(x)
$$

equivalently $\psi(x, t)$ satisfies the abstract Schrödinger equation (Jauch [35])

$$
i \hbar \frac{\partial \psi}{\partial t}(x, t)=\widehat{H} \psi(x, t) .
$$

We now make the following crucial assumption, which extrapolates to the general case what we have done for Hamiltonians of the type classical type "kinetic energy plus potential": the quantum dynamics is again given by the Kerner-Sutcliffe propagator (35) for small times $t$, i.e.,

$$
K\left(x, x^{\prime}, t\right)=\bar{K}\left(x, x^{\prime}, t\right)+O\left(t^{2}\right)
$$

the approximate propagator being given by

$$
\bar{K}\left(x, x^{\prime}, t\right)=\left(\frac{1}{2 \pi \hbar}\right)^{n} \int e^{\frac{i}{\hbar}\left(p\left(x-x^{\prime}\right)-\bar{H}\left(x, x^{\prime}\right) t\right)} d^{n} p
$$

where $\bar{H}$ is this time the averaged Hamiltonian function

$$
\bar{H}\left(x, x^{\prime}, p\right)=\int_{0}^{1} H\left(\tau x+(1-\tau) x^{\prime}, p\right) d \tau .
$$

Obviously, when $H=H_{\text {free }}+V$ the function $\bar{H}$ reduces to the function $H_{\text {free }}+\bar{V}$ considered in Section 2.

This assumption can be motivated as follows (see de Gosson [12], Proposition 15, §4.4). Let

$$
S\left(x, x^{\prime}, t\right)=\int_{\gamma} p d x-H d t
$$

be Hamilton's two-point function calculated along the phase space path leading from an initial point $\left(x^{\prime}, p^{\prime}, 0\right)$ to a final point $(x, p, t)$ (the existence of such a function for small $t$ is guaranteed by Hamilton-Jacobi theory; see e.g., Arnol'd [36] or Goldstein [37]). That function satisfies the Hamilton-Jacobi equation

$$
\frac{\partial S}{\partial t}+H\left(x, \nabla_{x} S\right)=0
$$

One then shows that the function

$$
\bar{S}\left(x, x^{\prime}, t\right)=p\left(x-x^{\prime}\right)-\bar{H}\left(x, x^{\prime}, p\right) t
$$

where $p$ is the momentum at time $t$ is an approximation to $S\left(x, x^{\prime}, t\right)$, in fact

$$
\bar{S}\left(x, x^{\prime}, t\right)-S\left(x, x^{\prime}, t\right)=O\left(t^{2}\right) .
$$


Here is an example: Choose $H=\frac{1}{2} p^{2} x^{2}$ (we are assuming here $n=1$ ); then

$$
S\left(x, x^{\prime}, t\right)=\frac{\left(\ln \left(x / x^{\prime}\right)\right)^{2}}{2 t} .
$$

Using the formula

$$
\bar{H}\left(x, x^{\prime}, p\right)=\frac{1}{6} p^{2}\left(x^{2}+x x^{\prime}+x^{\prime 2}\right)
$$

one shows after some calculations involving the Hamiltonian equations for $H$ that

$$
\bar{S}\left(x, x^{\prime}, t\right)=\frac{\left(\ln \left(x / x^{\prime}\right)\right)^{2}}{2 t}+O\left(t^{2}\right)
$$

(see [12], Chapter 4, Examples 10 and 16 for detailed calculations).

We are now going to show that the operator $\widehat{H}$ can be explicitly and uniquely determined from the knowledge of $\bar{K}\left(x, x^{\prime}, t\right)$.

Theorem 4. If we assume that the short-time propagator is given by formula (51) then the operator $\hat{H}$ appearing in the abstract Schrödinger Equation (49) is given by

$$
\widehat{H} \psi(x)=\left(\frac{1}{2 \pi \hbar}\right)^{n} \int e^{\frac{i}{\hbar} p\left(x-x^{\prime}\right)} \bar{H}\left(x, x^{\prime}, p\right) \psi\left(x^{\prime}\right) d^{n} p d^{n} x^{\prime} .
$$

Proof. Differentiating both sides of the equality (47) with respect to time we get

$$
i \hbar \frac{\partial \psi}{\partial t}(x, t)=i \hbar \int \frac{\partial K}{\partial t}\left(x, x^{\prime}, t\right) \psi_{0}\left(x^{\prime}\right) d^{n} x^{\prime}
$$

since $K$ itself satisfies the Schrödinger Equation (49) we thus have

$$
\widehat{H} \psi(x, t)=i \hbar \int \frac{\partial K}{\partial t}\left(x, x^{\prime}, t\right) \psi_{0}\left(x^{\prime}\right) d^{n} x^{\prime} .
$$

It follows, using the assumptions (50) and (51), that

$$
\widehat{H} \psi(x, t)=i \hbar \int \frac{\partial \bar{K}}{\partial t}\left(x, x^{\prime}, t\right) \psi_{0}\left(x^{\prime}\right) d^{n} x^{\prime}+O(t)
$$

and hence, letting $t \rightarrow 0$,

$$
\widehat{H} \psi_{0}(x)=i \hbar \int \frac{\partial \bar{K}}{\partial t}\left(x, x^{\prime}, 0\right) \psi_{0}\left(x^{\prime}\right) d^{n} x^{\prime}
$$

Introducing the notation

$$
\bar{S}\left(x, x^{\prime}, t\right)=p\left(x-x^{\prime}\right)-\bar{H}\left(x, x^{\prime}, p\right) t
$$

we have

$$
\begin{aligned}
\frac{\partial \bar{K}}{\partial t}\left(x, x^{\prime}, t\right) & =\left(\frac{1}{2 \pi \hbar}\right)^{n} \frac{i}{\hbar} \int e^{\frac{i}{\hbar} \bar{S}\left(x, x^{\prime}, t\right)} \frac{\partial \bar{S}}{\partial t}\left(x, x^{\prime}, t\right) d^{n} p^{\prime} \\
& =\left(\frac{1}{2 \pi \hbar}\right)^{n} \frac{1}{i \hbar} \int e^{\frac{i}{\hbar} \bar{S}\left(x, x^{\prime}, t\right)} \bar{H}\left(x, x^{\prime}, p^{\prime}\right) d^{n} p^{\prime}
\end{aligned}
$$

Taking the limit $t \rightarrow 0$ and multiplying both sides of this equality by it we finally get

$$
\widehat{H} \psi_{0}(x)=\left(\frac{1}{2 \pi \hbar}\right)^{n} \int e^{\frac{i}{\hbar} p\left(x-x^{\prime}\right)} \bar{H}\left(x, x^{\prime}, p^{\prime}, t^{\prime}\right) \psi_{0}\left(x^{\prime}\right) d^{n} p^{\prime} d^{n} x^{\prime}
$$

which proves (53). 
We will call the operator $\widehat{H}$ defined by (53) the Born-Jordan quantization of the Hamiltonian function $H$. That this terminology is justified is motivated below.

\subsection{The Case of Monomials}

Let us show that (53) reduces to the usual Born-Jordan quantization rule (1) when $H=x^{m} p^{\ell}$ (we are thus assuming dimension $n=1$ ). We have here

$$
H\left(\tau x+(1-\tau) x^{\prime}, p\right)=\left(\tau x+(1-\tau) x^{\prime}\right)^{m} p^{\ell}
$$

hence, using the binomial formula,

$$
H\left(\tau x+(1-\tau) x^{\prime}, p\right)=\sum_{k=0}^{m}\left(\begin{array}{l}
m \\
k
\end{array}\right) \tau^{k}(1-\tau)^{m-k} x^{k} p^{\ell} x^{\prime m-k}
$$

Integrating from 0 to 1 in $\tau$ and noting that

$$
\int_{0}^{1} \tau^{k}(1-\tau)^{m-k} d \tau=\frac{k !(m-k) !}{(m+1) !}
$$

we get

$$
\bar{H}\left(x, x^{\prime}, p\right)=\frac{1}{m+1} \sum_{k=0}^{m} x^{k} p^{\ell} x^{\prime m-k}
$$

and hence, using the definition (53) of $\widehat{H}$,

$$
\begin{aligned}
\widehat{H} \psi(x) & =\frac{1}{2 \pi \hbar(m+1)} \sum_{k=0}^{m} \int_{-\infty}^{\infty} e^{\frac{i}{\hbar} p\left(x-x^{\prime}\right)} x^{k} p^{\ell} x^{\prime m-k} \psi\left(x^{\prime}\right) d p d x^{\prime} \\
& =\frac{x^{k}}{2 \pi \hbar(m+1)} \sum_{k=0}^{m} \int_{-\infty}^{\infty}\left(\int_{-\infty}^{\infty} e^{\frac{i}{\hbar} p\left(x-x^{\prime}\right)} p^{\ell} d p\right) x^{\prime m-k} \psi\left(x^{\prime}\right) d x^{\prime}
\end{aligned}
$$

In view of the Fourier inversion Formula (34) we have

$$
\frac{1}{2 \pi \hbar} \int_{-\infty}^{\infty} e^{\frac{i}{\hbar} p\left(x-x^{\prime}\right)} p^{\ell} d p=(-i \hbar)^{\ell} \delta^{(\ell)}\left(x-x^{\prime}\right)
$$

so that we finally get

$$
\widehat{H} \psi(x)=\frac{1}{m+1} \sum_{k=0}^{m} x^{k}(-i \hbar)^{\ell} \frac{\partial^{\ell}}{\partial x^{\ell}}\left(x^{m-k} \psi\right),
$$

which is equivalent to (1) since $\hat{p}^{\ell}=(-i \hbar)^{\ell} \partial^{\ell} / \partial x^{\ell}$.

\subsection{Physical Hamiltonians}

Let us now show that the Born-Jordan quantization of a physical Hamiltonian of the type

$$
H=\sum_{j=1}^{n} \frac{1}{2 m_{j}}\left(p_{j}-A_{j}(x)\right)^{2}+V(x)
$$

coincide with the usual operator

$$
\widehat{H}=\sum_{j=1}^{n} \frac{1}{2 m_{j}}\left(-i \hbar \frac{\partial}{\partial x_{j}}-A_{j}(x)\right)^{2}+V(x)
$$


obtained by Weyl quantization (the functions $A_{j}$ and $V$ are assumed to be $C^{1}$ ). Since the quantizations of $p_{j}^{2}, A_{j}(x)$ and $V(x)$ are the same in all quantization schemes (they are respectively $-\hbar^{2} \partial^{2} / \partial x_{j}^{2}$ and multiplication by $A_{j}(x)$ and $V(x)$ ), we only need to bother about the cross-products $p_{j} A(x)$. We claim that

$$
\widehat{p_{j} A} \psi=-\frac{i \hbar}{2}\left[\frac{\partial}{\partial x_{j}}(A \leftarrow)+A \frac{\partial \psi}{\partial x_{j}}\right]
$$

from which (58) immediately follows. Let us prove (59); it is sufficient to do this in the case $n=1$. Denoting by $\overline{p A}$ the Born-Jordan quantization of the function $p A$ we have

$$
\overline{p A}\left(x, x^{\prime}, p\right)=p \int_{0}^{1} A\left(\tau x+(1-\tau) x^{\prime}\right) d \tau=p \bar{A}\left(x, x^{\prime}\right)
$$

and hence

$$
\begin{aligned}
\widehat{p A} \psi(x) & =\frac{1}{2 \pi \hbar} \int e^{\frac{i}{\hbar} p\left(x-x^{\prime}\right)} p \bar{A}\left(x, x^{\prime}\right) \psi\left(x^{\prime}\right) d x^{\prime} d p \\
& =\int_{-\infty}^{\infty}\left(\frac{1}{2 \pi \hbar} \int_{-\infty}^{\infty} e^{\frac{i}{\hbar} p\left(x-x^{\prime}\right)} p d p\right) \bar{A}\left(x, x^{\prime}\right) \psi\left(x^{\prime}\right) d x^{\prime} .
\end{aligned}
$$

In view of (34) the expression between the square brackets is $-i \hbar \delta^{\prime}\left(x-x^{\prime}\right)$ so that

$$
\begin{aligned}
\widehat{p A} \psi(x) & =-i \hbar \int_{-\infty}^{\infty} \delta^{\prime}\left(x-x^{\prime}\right) \bar{A}\left(x, x^{\prime}\right) \psi\left(x^{\prime}\right) d x^{\prime} \\
& =-i \hbar \int_{-\infty}^{\infty} \delta\left(x-x^{\prime}\right) \frac{\partial}{\partial x^{\prime}}\left(\bar{A}\left(x, x^{\prime}\right) \psi\left(x^{\prime}\right)\right) d x^{\prime} \\
& \left.\left.=-i \hbar\left(\frac{\partial \bar{A}}{\partial x^{\prime}}(x, x) \psi(x)\right)+\bar{A}(x, x) \frac{\partial \psi}{\partial x^{\prime}}(x)\right)\right)
\end{aligned}
$$

Now, by definition of $\bar{A}\left(x, x^{\prime}\right)$ we have $\bar{A}(x, x)=A(x)$ and

$$
\frac{\partial \bar{A}}{\partial x^{\prime}}(x, x)=\int_{0}^{1}(1-\tau) \frac{\partial A}{\partial x}(x) d \tau=\frac{1}{2} \frac{\partial A}{\partial x}(x)
$$

and hence

$$
\widehat{p A} \psi=-\frac{i \hbar}{2} \frac{\partial A}{\partial x} \psi-i \hbar A \frac{\partial \psi}{\partial x}
$$

which is the same thing as (59).

\section{Discussion}

Both Kerner and Sutcliffe, and Cohen relied on path integral arguments which were doomed to fail because of the multiple possible choices of histories in path integration. However, it follows from our rigorous constructions that Kerner and Sutcliffe's insight was right, even though their construction was not rigorously mathematically justified. While there is, as pointed out by Cohen [22], a great latitude in choosing the short-time propagator, thus leading to different quantizations, our argument did not make use of any path-integral argument; what we did was to propose a short-time propagator which is exact up to order $O\left(t^{2}\right)$ (as opposed to those obtained by using midpoint rules), and to show that if one use this propagator, then one must quantize Hamiltonian functions (and in particular monomials) following the prescription proposed by Born and Jordan in the case of monomials.

Funding: This work has been financed by the Grant P 27773-N25 of Austrian Science Fund (FWF).

Conflicts of Interest: The author declares no conflict of interest. 


\section{References}

1. Dewey, T.G. Numerical mathematics of Feynman path integrals and the operator ordering problem. Phys. Rev. A 1990, 42, 32-37. [CrossRef] [PubMed]

2. Hall, M. Weyl's rule and Wigner equivalents for phase space monomials. J. Phys. A Math. Gen. 1985, 18, $29-36$. [CrossRef]

3. Khandekar, D.C.; Lawande, S.V. Feynman Path Integrals: Some Exact Results and Applications. Phys. Reps. 1986, 137, 115-229. [CrossRef]

4. Kumano-go, N.; Fujiwara, D. Phase space Feynman path integrals via piecewise bicharacteristic paths and their semiclassical approximations, Bull. Sci. Math. 2008, 132, 313-357.

5. Mayes, I.W.; Dowker, J.S. Canonical functional integrals in general coordinates. Proc. R. Soc. Lond. A 1972, 327, 131-135. [CrossRef]

6. Mayes, I.W.; Dowker, J.S. Hamiltonian orderings and functional integrals. J. Math. Phys. 1973, 14, $434-439$. [CrossRef]

7. Shewell, J.R. On the Formation of Quantum-Mechanical Operators. Am. J. Phys. 1959, 27, 16-21. [CrossRef]

8. de Gosson, M. Born-Jordan Quantization and the Equivalence of the Schrödinger and Heisenberg Pictures. Found. Phys. 2014, 44, 1096-1106. [CrossRef] [PubMed]

9. McCoy, N.H. On the function in quantum mechanics which corresponds to a given function in classical mechanics. Proc. Natl. Acad. Sci. USA 1932, 18, 674-676. [CrossRef] [PubMed]

10. Dahl, J.P.; Springborg, M. Wigner's phase space function and atomic structure: I. The hydrogen atom ground state. Mol. Phys. 1982, 47, 1001-1019. [CrossRef]

11. de Gosson, M. The Angular Momentum Dilemma and Born-Jordan Quantization. Found. Phys. 2017, 47, 61-70. [CrossRef]

12. de Gosson, M. Born-Jordan Quantization: Theory and Applications; Springer: New York, NY, USA, 2016.

13. Heisenberg, W. Über quantentheoretische Umdeutung kinematischer und mechanischer Beziehungen. $Z$. Physik 1925, 33, 879-893. [CrossRef]

14. Born, M.; Jordan, P. Zur Quantenmechanik. Z. Physik 1925, 34, 858-888. [CrossRef]

15. Born, M.; Heisenberg, W.; Jordan, P. Zur Quantenmechanik II. Z. Physik 1925, 35, 557-615. [CrossRef]

16. Kerner, E.H.; Sutcliffe, W.G. Unique Hamiltonian Operators via Feynman Path Integrals. J. Math. Phys. 1970, 11, 391-393. [CrossRef]

17. Garrod, C. Hamiltonian Path-Integral Methods. Rev. Mod. Phys. 1966, 38, 483-494. [CrossRef]

18. Kauffmann, S.K. Unique Closed-Form Quantization Via Generalized Path Integrals or by Natural Extension of the Standard Canonical Recipe. arXiv 1995, arXiv:hep-th/9505189v2.

19. Kauffmann, S.K. Unambiguous Quantization from the Maximum Classical Correspondence that Is Self-consistent: The Slightly Stronger Canonical Commutation Rule Dirac Missed. Found. Phys. 2011, 41, 805-819. [CrossRef]

20. de Gosson, M. Symplectic Covariance properties for Shubin and Born-Jordan pseudo-differential operators. Trans. Amer. Math. Soc. 2013, 365, 3287-3307. [CrossRef]

21. de Gosson, M.; Luef, F. Preferred Quantization Rules: Born-Jordan vs. Weyl; Applications to Phase Space Quantization. J. Pseudo-Differ. Oper. Appl. 2011, 2, 115-139. [CrossRef]

22. Cohen, L. Hamiltonian Operators via Feynman Path Integrals. J. Math. Phys. 1970, 11, 3296-3297. [CrossRef]

23. Makri, N.; Miller, W.H. Correct short time propagator for Feynman path integration by power series expansion in $\Delta t$. Chem. Phys. Lett. 1988, 15, 1-8. [CrossRef]

24. Makri, N.; Miller, W.H. Exponential power series expansion for the quantum time evolution operator. J. Chem. Phys. 1989, 90, 904-911. [CrossRef]

25. de Gosson, M. The Principles of Newtonian and Quantum Mechanics, the Need for Planck's Constant $\hbar$; Imperial College Press: London, UK, 2001.

26. Gutzwiller, M.C. Chaos in Classical and Quantum Mechanics; Springer Science \& Business Media: Berlin/Heidelberg, Germany, 2013.

27. Schulman, L.S. Techniques and Applications of Path Integration; Wiley: New York, NY, USA, 1981.

28. Maslov, V.P.; Fedoriuk, M.V. Semi-Classical Approximation in Quantum Mechanics; Springer Science \& Business Media: Berlin/Heidelberg, Germany, 2001; Volume 7. 
29. de Gosson, M. Symplectic Methods in Harmonic Analysis and in Mathematical Physics; Springer Science \& Business Media: Berlin/Heidelberg, Germany, 2011.

30. de Gosson, M.; Hiley, B.J. Short-time quantum propagator and Bohmian trajectories. Phys. Lett. A 2013, 377 , 3005-3008. [CrossRef] [PubMed]

31. de Gosson, M.; Hiley, B.J. Hamiltonian flows, short-time quantum propagators and the quantum Zeno effect. J. Phys. Conf. Ser. 2013, 504, 012027. [CrossRef]

32. Park, D. Introduction to the Quantum Theory; McGraw-Hill Inc.: New York, NY, USA, 1992.

33. Hörmander, L. The Analysis of Linear Partial Differential Operators I; Springer: New York, NY, USA, 1985; Volume 256.

34. Stone, M.H. Linear Transformations in Hilbert Space: III. Operational Methods and Group Theory. Proc. Natl. Acad. Sci. USA 1930, 16, 172-175. [CrossRef] [PubMed]

35. Jauch, J.M.; Morrow, R.A. Foundations of quantum mechanics. Am. J. Phys. 1968, 36, 771. [CrossRef]

36. Arnold, V.I. Mathematical Methods of Classical Mechanics, Graduate Texts in Mathematics, 2nd ed.; Springer: New York, NY, USA, 1989.

37. Goldstein, H. Classical Mechanics, 2nd ed.; Addison-Wesley: Boston, MA, USA, 1980.

(C) 2018 by the author. Licensee MDPI, Basel, Switzerland. This article is an open access article distributed under the terms and conditions of the Creative Commons Attribution (CC BY) license (http:/ / creativecommons.org/licenses/by/4.0/). 